\title{
A Systematic Sensitivity Analysis of the Performance of a Transcutaneous Energy Transmitter for Design Purposes
}

\author{
Daniela Wolter Ferreira, Luiz Lebensztajn, \\ Departamento de Engenharia de Energia e Automação Elétricas, Escola Politécnica da Universidade de São \\ Paulo, São Paulo, Brasil-daniwolter@usp.br, leb@pea.usp.br
}

\begin{abstract}
This work studies a Transcutaneous Energy Transmitter (TET) which uses electromagnetic fields to transfer power from outside the body to an artificial organ in the body, working like a high frequency transformer with the skin being part of the magnetic coupling between the primary (external coil) and secondary (internal coil). For this reason, this project made two different kinds of analyses of the efficiency and the induced current density in a biologic tissue: first, simulations were performed using core types of distinct geometries, each with multiples sizes, ranking which type is more recommended; second, the sensitivity of the chosen core type to misalignments was evaluated for different dimensions. In addition, the sensitivity to the core permeability was also investigated for both analyses.
\end{abstract}

Index Terms-Finite Element Method, Induced Current Density, Transcutaneous Energy Transmitter.

\section{INTRODUCTION}

The advancement of medicine spawned many artificial organs that are mostly or completely implanted inside the body. Such organs require the supply of power internally to the body, bringing more complexity to the complete system and often forcing the patients to have wires crossing their skin, what can cause infections and discomfort. Trying to reduce these complexities, several researchers widely study options to transfer electric energy to an internal electrical subsystem of direct current (DC) that provides power to these artificial organs and their internal backup battery [1][3]. This technology, called Transcutaneous Energy Transmission, or TET, normally works through inductive link similar to a transformer with the primary and secondary windings placed above and below the skin, respectively [4].

However, the skin between the windings can vary in thickness more than $5 \mathrm{~mm}$, creating low coupling and considerable high induced currents, and thus requiring higher voltage from the external power supply and working at low efficiency.

Therefore, a proper design of this system will allow obtaining a highly efficient system with fewer losses that can be used in several different implanted artificial organs projects. During the designing phase, the engineer deals with several important decisions with the core geometry and size being two of them: "what is the best core geometry and size that would instigate the least discomfort to the patient without losing reliability at unsteady situations?" This paper introduces analyses of different 
TET project parameters, showing how the performance of the TET is affected with the different sizes, geometries and core permeability of the TET.

The paper is organized as follows: In Section II, the transcutaneous energy transmitter system is briefly presented with the collateral effect that it may cause. In Sections III, the methodology is described, pointing out the different simulated core types. Afterwards, the achieved results are analyzed in Section IV, which is divided in the two steps of methodology: evaluation of the core type with aligned coils; and evaluation of the sensitivity of the chosen core type to misalignments. Finally, the conclusion is drawn in Section V.

\section{TRANSCUTANEOUS ENERGY TRANSMITTER SYSTEM AND ITS EFFECTS ON BIOLOGICAL TISSUES}

\section{A. Transcutaneous Energy Transmitter}

Most of the studied proposals of TET development are based on the principle of electromagnetic field, transferring energy wirelessly from an external coil to a coil inside the body. It is similar to a transformer with the primary coil outside the body transmitting power to a secondary coil inside the body and having the skin and fat as part of the inductive link between the coils. Thus, the coils form a weakly coupled transformer as they are not connected by a common core and they could be misaligned during any small movement from the patient. For this reason, in this work, the primary and secondary coils and their respective cores are considered a single element called transformer.

It was already presented in [5] that the coils should be distributing their turns across the radii instead of concentrating them at the outer circumferences. Thus, in this paper, the external and internal coils and cores constitute a planar transformer.

The medium between the transmitting and receiving systems contains the skin and could also contain fat, of which the dielectric properties vary with the electromagnetic wave frequency as collected by [6]. Since these tissues vary their thickness from person to person, the gap between the transmitter and the receiver can vary from 5 to $30 \mathrm{~mm}$, what characterize the TET as a transformer with a large gap.

\section{B. Electromagnetic Effects on Biological Tissues}

Since the biological tissue in between the inductive link has a small conductivity, currents may be induced due to the process of transmission. These currents can stimulate neural or muscle excitation or actions as it has been researched for MRI (Magnetic Resonant Images) [7]. Thus, the restriction of the induced current magnitude flowing through a surface of the biological tissue can minimize these non-desired effects. This induced current density is expressed in Ampere per square meter $\left(\mathrm{A} / \mathrm{m}^{2}\right)$ as:

$$
J=\sigma . E
$$

where $\mathrm{E}$ is the root mean square (RMS) electric field (V/m); and $\sigma$ is the electrical conductivity of the biological tissue $(\mathrm{S} / \mathrm{m})$.

There is intense discussion in this regard trying to establish limits for such effects. In Brazil, the government follows the World Health Organization (WHO) that recommends the limit established by 
the International Commission on Non-Ionizing Radiation Protection (ICNIRP) [8]. According to ICNIRP rules, a patient that knows the risk of the exposure to this magnetic field and is oriented to follow the right procedure to minimize the risks can be exposed at a maximum value of the induced RMS current density in $\mathrm{mA} / \mathrm{m}^{2}$ not exceeding the value of the frequency divided by one hundred, i.e., $J_{\max }=f / 100$.

Therefore, this paper works with the concept of relative current density in the skin in percentage, which is the induced current density divided by the limit of it at the frequency of the induction and multiplied by one hundred $\left(J / J_{\max } \cdot 100 \%\right)$. Since the acceptable limit by ICNIRP is the frequency divided by 100 , the relative current density is the induced current in $\mathrm{mA} / \mathrm{m}^{2}$ divided by the frequency and multiplied by 10000 .

\section{METHODOLOGY}

The main idea of this paper is to explore the TET projects ability to provide more comfort and reliability to the patient. But how can someone measure comfort or stability? Someone could say that discomfort is measured by anything that takes the patient out of his routine, like carrying a heavy weight or big object. Regarding the stability, the TET is considered reliable if it functions at comparable performance even in uncommon situations, such as an unusual position as when the primary and the secondary coils are misaligned.

In this way, during the design phase of the TET, the engineer should seek to maximize efficiency and/or minimize the volume or weight of the TET, taking in consideration the undesirable conditions to which the TET could be subjected and the constraints of the project - relative induced current density in the biological tissue and secondary output voltage within the required range.

Since part of the TET will be is positioned in the body of the patient, it will be subjected to different kind of coupling situations, such as:

- Distance between the coils - the skin thickness could vary according to the patient.

- Distance between the axes of the coils - since the secondary coil is position inside the body, the alignment between the coils is very hard to achieve.

The parameters of the TET (degrees of freedom) that could help to provide the requirements of this project (maximum efficiency, minimum volume, low relative induced current density) could be:

- Parameters related to the magnetic properties, such as number of turns as well as relative permeability of the ferromagnetic material, internal and external diameters, and geometry of the cores.

- Parameters related to the electric system, such as applied voltage and frequency.

In this work, different simulations were performed using Finite Element Method (FEM) through Flux software [9]. In Flux, the geometry considering magnetic cores (gray), copper coils (green), skin (pink), fat (orange) and air (blue) was drawn as shown in Fig. 1. Observe that the primary core, 
external to the body, is involved by air while the secondary core, underneath the skin, is involved by fat. The thickness of the simulated skin was considered to be $5 \mathrm{~mm}$, thus the gap between primary and secondary coils is $5 \mathrm{~mm}$. The simulations considered the electrical and magnetic properties of the skin, fat, copper coils and magnetic cores, as will be explained in the following pages.

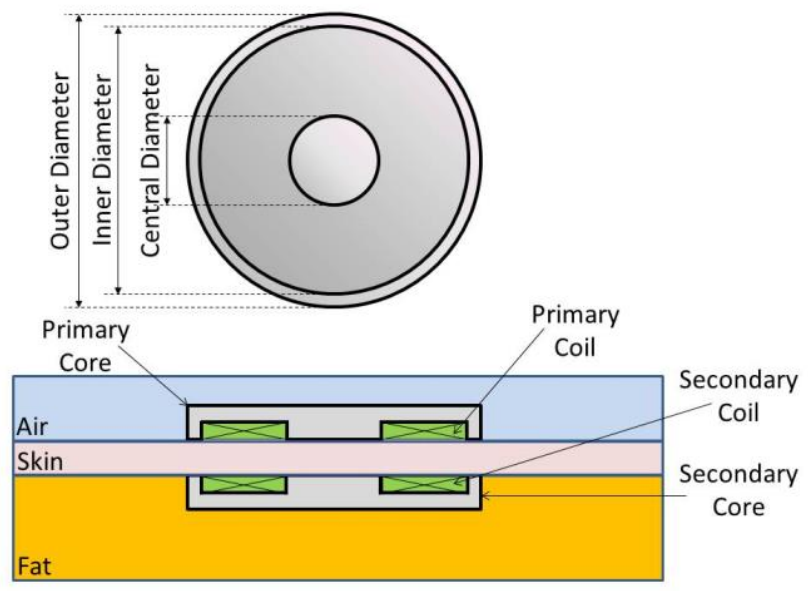

Fig. 1. Geometry drawn in Flux.

First, the TET was implemented in Flux2D considering the five different geometries of core shown in Fig. 2 plus coreless coils, i.e., the coils with no magnetic core around. The Flux2D software performed a set of simulations with the coils aligned for the range of dimensions shown in Table I. All the geometrical parameters are shown in Fig. 1.

TABLE I. DIMENSIONS WITH THEIR DEFAULT VALUES AND RANGE OF SIMULATION

\begin{tabular}{ccc}
\hline Dimensions & Default & Range \\
\hline Frequency & $100 \mathrm{kHz}$ & {$\left[\begin{array}{ll}50 & 300\end{array}\right] \mathrm{kHz}$} \\
Outer Core Diameter & $50 \mathrm{~mm}$ & {$\left[\begin{array}{ll}45 & 70\end{array}\right] \mathrm{mm}$} \\
Inner Core Diameter & $40 \mathrm{~mm}$ & {$\left[\begin{array}{ll}20 & 45\end{array}\right] \mathrm{mm}$} \\
Central Core Diameter & $10 \mathrm{~mm}$ & {$\left[\begin{array}{ll}8 & 24\end{array}\right] \mathrm{mm}$} \\
Primary wire turns & 23 turns & {$\left[\begin{array}{ll}23 & 45\end{array}\right]$ turns } \\
Secondary wire turns & 45 turns & {$\left[\begin{array}{ll}23 & 45\end{array}\right]$ turns } \\
Relative Core Permeability & 3400 & {$[24000]$} \\
\hline
\end{tabular}

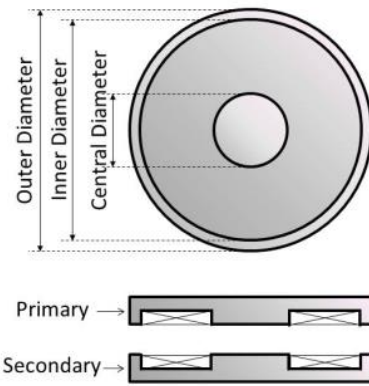

(I)

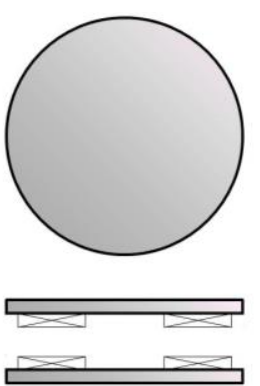

(II)

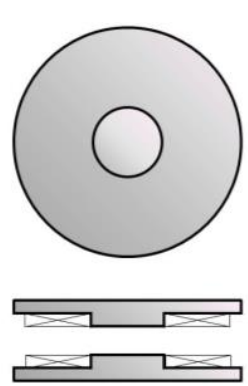

(III)

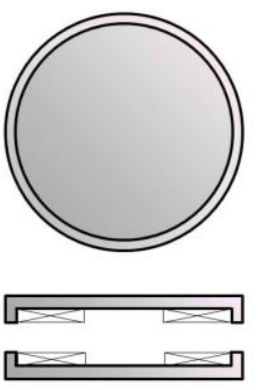

(IV)

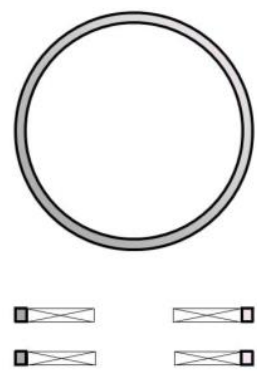

(V)

Fig. 2. Different geometries of the TET.

Note that all the analyzed geometries are circular with small differences. A circular core was used 
due to the shape of the coil winding and because sharp corners could hurt the patient, what would directly affect his comfort. Following this trend of thought, the more round the core geometry is, the more comfortable the TET is for the patient. Furthermore, a square coil would add an instability problem to the system in regards to misalignment. Considering that the central axes are lined up, a round core is completely aligned at any rotational position of the cores. In addition, when the axes are displaced, the TET behaves similarly at any direction of displacement due to the symmetry. A square core, on the other hand, has symmetry only if the relative rotational angle between the cores is a multiple of $90^{\circ}$. Thus, the behavior of this type of TET with displacements is different if the core axes displace in different directions. Also, even if the central axes are lined up, a square TET would change its behavior at any rotation different than $90^{\circ}$.

It is important to remark that the volume described by the area between the inner diameter and central diameter is the space reserved to insert the coil. All the simulations in Flux were performed considering that stranded copper coils AWG 28 fill this whole space independently of the number of turns. That means that the gap between the turns is variable and depending on the available space between the inner and central diameter and the number of turns. With less turns and bigger area, the turns are less tight together.

Thus, the resistance of the electrical stranded coil conductor coupled with the FEM geometry regions on Flux depends on the length, the cross-section and the resistivity of the massive wire AWG 28. The length of the wire $(l)$ was calculated based on the number of turns $(N)$ and considering the average between the inner $\left(D_{\text {inner }}\right)$ and central $\left(D_{\text {central }}\right)$ diameter, i.e.

$$
l=N . \pi \cdot\left(\frac{D_{\text {central }}+D_{\text {inner }}}{2}\right)
$$

The electrical properties of the skin between each core and the fat around the secondary core, defined by [6] as shown in Table II, were used to account for their losses during the simulation of the system.

TABLE II. ELECTRICAL PROPERTIES OF THE BIOLOGICAL TISSUES (WET SKIN AND FAT) USED TO COMPUTE THE CURRENT DENSITY AND EFFICIENCY

\begin{tabular}{ccccc}
\hline Tissue & Frequency [Hz] & Conductivity [S/m] & Relative permittivity & Loss tangent \\
\hline \multirow{6}{*}{ Wet Skin } & 10000 & 0.002932 & 29010 & 0.18166 \\
& 100000 & 0.065836 & 15357 & 0.77063 \\
& 150000 & 0.093995 & 11362 & 0.99141 \\
& 200000 & 0.11502 & 8849.2 & 1.1682 \\
& 250000 & 0.13116 & 7180.1 & 1.3135 \\
& 300000 & 0.14402 & 6012.6 & 1.4352 \\
\multirow{6}{*}{ Fat } & 10000 & 0.02383 & 1085.3 & 39.469 \\
& 100000 & 0.024414 & 92.885 & 47.247 \\
& 150000 & 0.024513 & 68.11 & 43.131 \\
& 200000 & 0.024585 & 56.015 & 39.448 \\
& 250000 & 0.024642 & 48.837 & 36.281 \\
& 300000 & 0.02469 & 44.075 & 33.565 \\
\hline
\end{tabular}


Other losses considered in these simulations were the resistivity of the copper wire and the iron losses in the ferromagnetic core. In fact, in order to evaluate the performance of the TET, the ferromagnetic material was also modeled with different values of relative permeability in the range shown in Table I.

After the analysis of the 2D simulation, the chosen geometry type was implemented in Flux3D in order to make a new set of simulations with the coils misaligned for all the range of dimensions described in Table I.

In both sets of simulations, the variation of the configurations was done by varying individually each dimension in its range while keeping the other dimensions with the default value.

\section{RESULTS}

\section{A. Evaluating geometry type and size when coils are aligned}

The simulation of the different core types was performed using Flux2D with both coils aligned while varying the geometry according to Table I. The results are explicit in Fig. 3, which shows the efficiency and the relative current density as functions of each dimension for all core types.

Note that the worst configuration is when the coil has no magnetic core - the relative current density is the highest and the efficiency is one of the smallest. The configuration with core type $\mathrm{V}$ is as bad as coreless, since this type of core entails in low efficiency (some cases even lower than coreless) and the relative current density induced in the skin from this kind of core is one of the worst.

From Fig. 3, the most recommended type of core is number I, which implies the best values of efficiency and lowest values of relative current density. All the other type of cores (II, III and IV) has similar performance with comparable values of efficiency for any size of geometry.

It is also possible to observe in Fig. 3 that when the coils are aligned, the efficiency improves for most of the core types while:

(a) Increasing the number of external turns.

(b) Decreasing the number of internal turns. Although for core type $\mathrm{V}$ and coreless coils, the efficiency increases a little bit up to 35 turns and then starts decreasing again, for the other types of core, the efficiency improves with less turns in the internal coil.

(c) Decreasing the size of the central core diameter. Observe that as the central core diameter tends to zero, core type I looks similar to core type IV. However there is a conceptual difference: making the central core diameter of core type I tend to zero means the windings must have central diameter tending to zero, what is very difficult to obtain. Core type IV has no core in the center of the windings, yet it has internal diameter different than zero. Thus, Fig. 3 shows that core type $\mathrm{I}$ is the best core type and has better results as the central core diameter is smaller. 

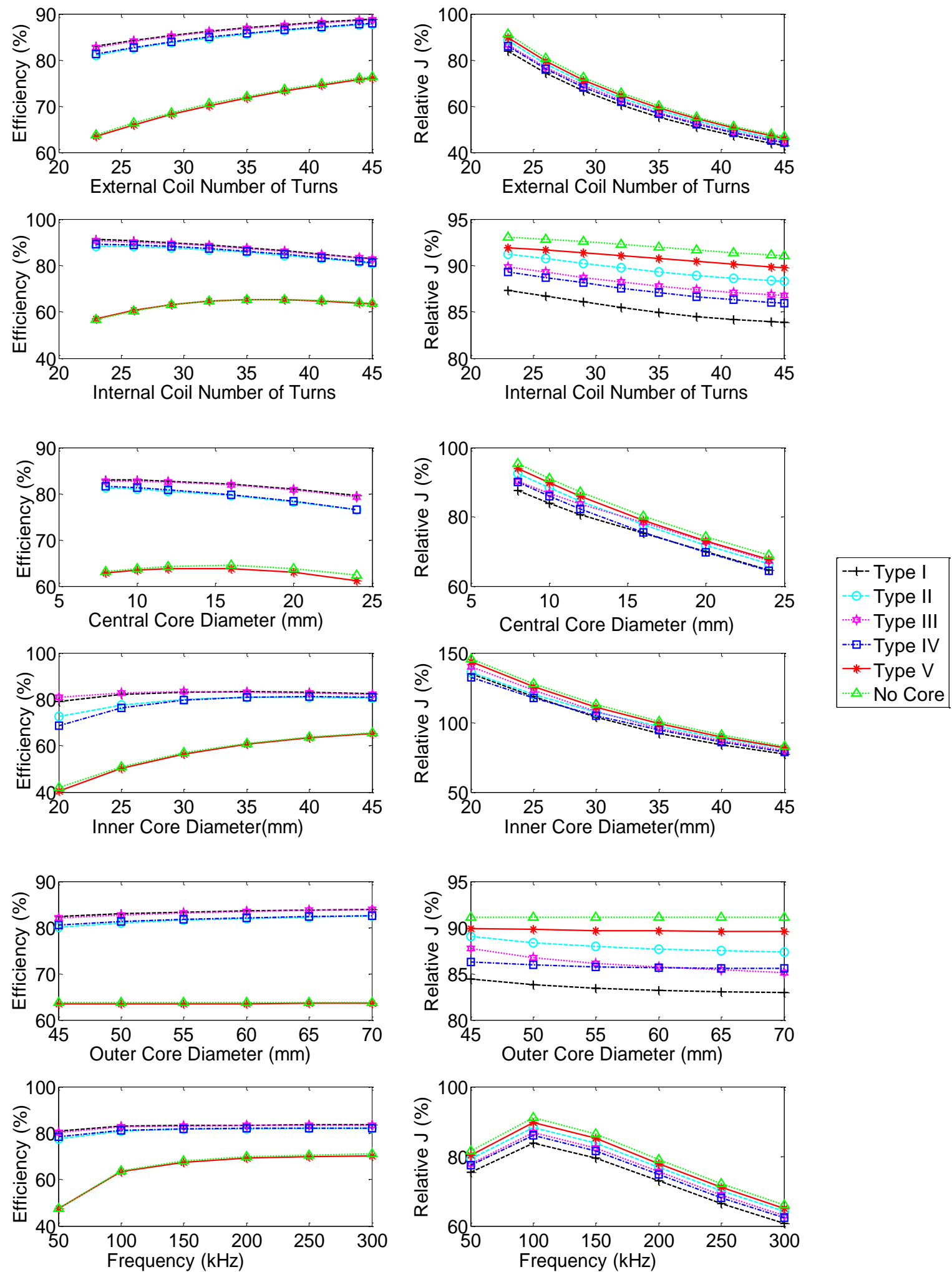

Fig. 3. Performance of TET with different geometry types and sizes. Each graphic is the variation of one of the parameters while the other parameters remain with the default value, i.e., frequency of $100 \mathrm{kHz}$, outer core diameter of $50 \mathrm{~mm}$, inner core diameter with $40 \mathrm{~mm}$, central core diameter with $10 \mathrm{~mm}$, internal coil with 45 turns and external coil with 23 turns. 
(d) Having the inner core diameter between $25 \mathrm{~mm}$ and $40 \mathrm{~mm}$ (though with values out of this range, the efficiency is not much worse). Note that for core type $\mathrm{V}$ and coreless coils, the bigger the inner core diameter is, the higher the efficiency is. The meaning of this parameter for coreless TET comes from the spacing between the turns of the coil. This observation shows that the wider the coreless coil is made, the more efficient power it will transfer.

(e) Increasing the outer core diameter. This parameter has no meaning for the coreless TET and very little influence on core type $\mathrm{V}$. This is the reason why, for those geometries, the efficiency and induced current are constant with the change of this parameter.

(f) Increasing the frequency. Though for frequencies higher than $100 \mathrm{kHz}$, the improvement of efficiency is negligible, the relative current density decreases at higher frequencies, having its worst value at $100 \mathrm{kHz}$ for the default configuration shown in Table I.

The behavior of the relative current density improves while:

(a) Increasing the number of external and internal turns.

(b) Increasing the size of the central and inner core diameter.

(c) Increasing the frequency, having its worst value when the frequency is $100 \mathrm{kHz}$. Here, there is a strong relation to the conductivity of the biologic tissues. As the frequency increases, the conductivity of the biologic tissues also increases, increasing the induced current. Looking at Table II, it is possible to notice that the increase rate of the tissue conductivity is smaller after $100 \mathrm{kHz}$. Since the limit allowed by ICNIRP increases linearly with frequency, the relative current density becomes smaller for frequencies at which the tissue conductivity increasing rate is smaller (at $100 \mathrm{kHz}$ ).

The variation of the outer core diameter did not change the value of the relative current density. Note that changing most of the parameter to improve the efficiency induces more current density in the body of the patient. That could suggest a multi-objective optimization, what is not the scope of this paper.

In addition to the core geometry, different core permeability was also analyzed for the different types of geometry. Similarly, the results identify the core type I as the recommended type. Moreover, Fig. 4 shows that the relative permeability of the material of the core affects very little the efficiency of the TET for values above 50, which means that the use of materials different than ferrite with lower relative permeability but still higher than 50 could be pertinent.

The behavior of the different core types with the variation of the thickness of the core was also analyzed, observing that the values of efficiency and relative current density were almost the same for all simulated thickness and the behavior with the change of core is similar to what is observed with the other dimensions - type I best results, type V worst results, and type II, III and IV similar results. 

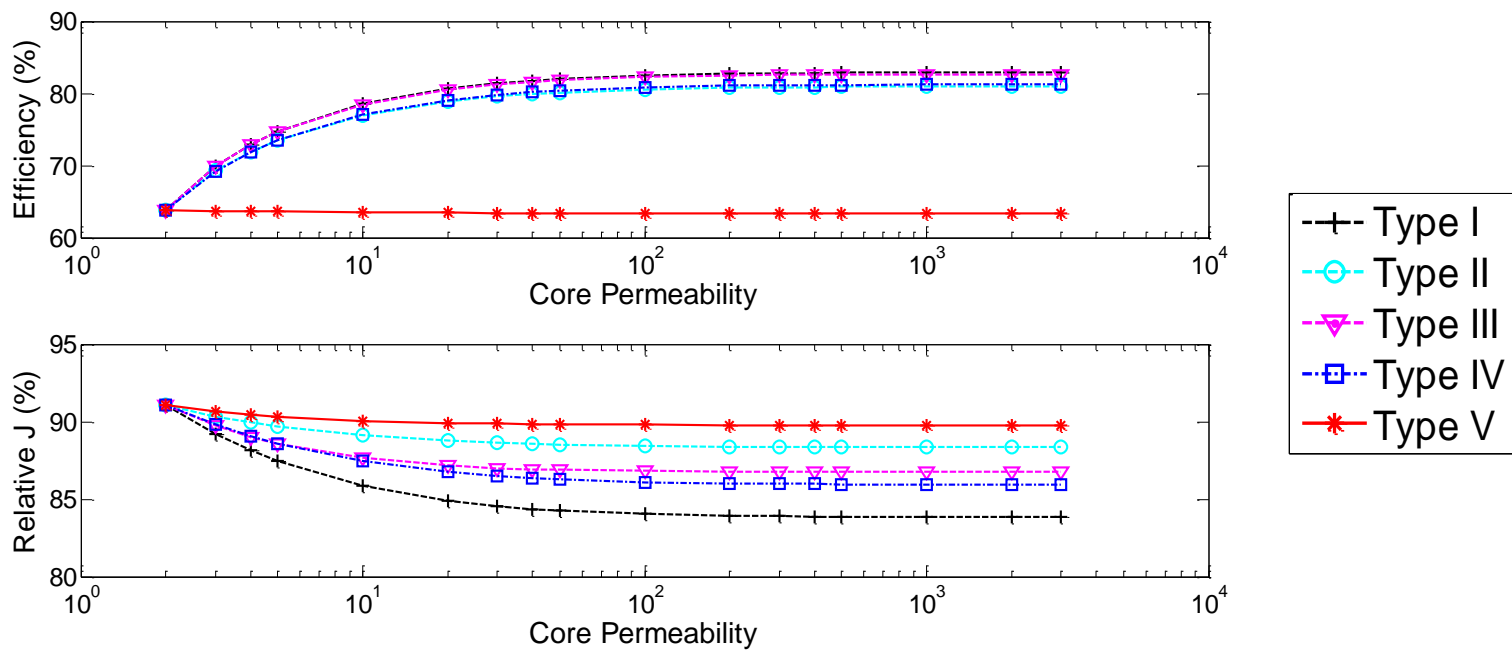

Fig. 4. Response of the TET with different core material and type.

The evaluation of efficiency and relative current density with variation of core permeability was performed by varying the permeability of both internal and external cores. Thus, the behavior of all core types might tend to the same when the relative permeability is tending to zero, what would be the configuration with coreless coils. Since type I was appointed as the best core, it was simulated with different relative permeability of each core, keeping the permeability of the other core constant at low value of 20, as shown in Fig. 5.
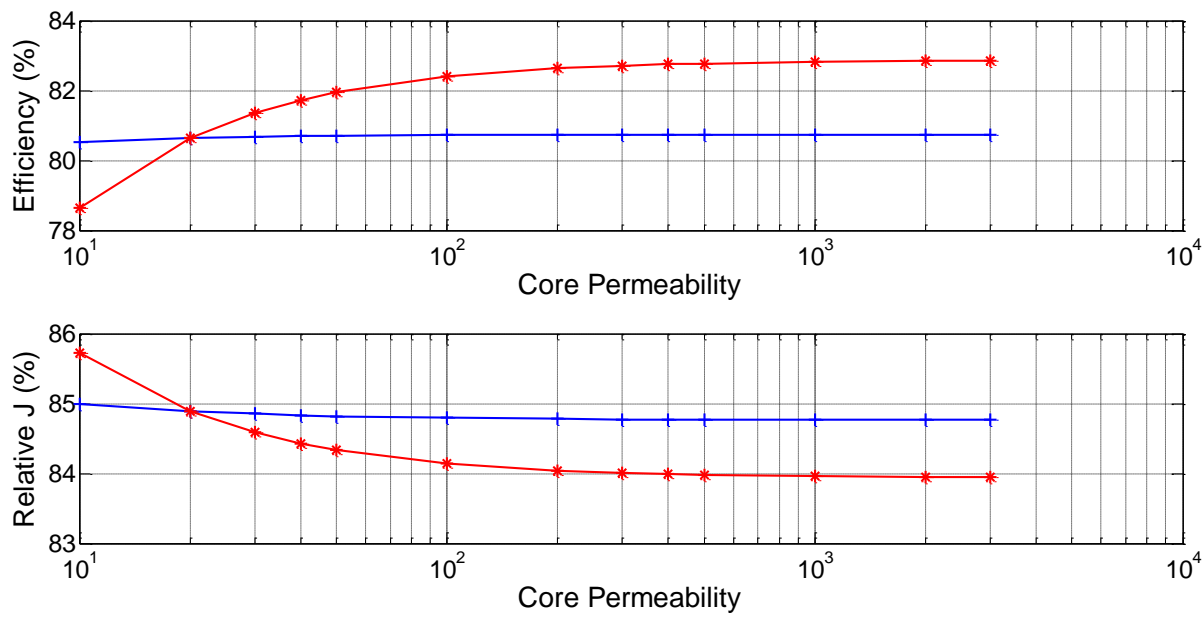

+ Internal Core Permeability Variation (External Core Permeability $=20$ )

$\rightarrow$ - External Core Permeability Variation (Internal Core Permeability $=20$ )

Fig. 5. Variation of the relative permeability only in one of the cores, keeping the other core with relative permeability $=20$ (Simulation only for type I)

Note that, when the external core relative permeability is constant at low value, the variation of the internal core relative permeability almost does not change the behavior of the system. However, when the internal core has low constant relative permeability, the curve of Fig. 5 is similar to the curve of type I from Fig. 4. That means that the effect observed in Fig. 4 is mainly due to the variations on the relative permeability of the external core. This observation suggests a configuration where the internal coil has very low permeability (eventually even no core) and the external coil has a core type I with 
permeability bigger than 50 .

\section{B. Evaluating stability with misalignment}

Seeking to analyze the behavior of the TET when the external coil is not aligned with the internal coil, simulations with core type I (the most recommended configuration from the previous item), 45 turns in the primary coil and 23 turns in the secondary coil were performed.

Fig. 6 shows the results of those simulations, presenting the response of the efficiency and relative current density for the misalignment at all the configurations in the range depicted by Table I. Observe that the default values from Table I are still the same except for the primary and secondary number of turns which are now 45 and 23, respectively.

It can be seen that, regardless of the change of the geometry size, the efficiency declines about $20 \%$ when the misalignment is up to $10 \mathrm{~mm}$ and then sinks to very low values for misalignments up to 20 $\mathrm{mm}$. By further increasing the misalignments, the efficiency slightly improves, stabilizing at similar values between 25 and $30 \mathrm{~mm}$. Above $30 \mathrm{~mm}$ of misalignment, the efficiency devaluates again.

The declination of the efficiency is strongly influenced by the size of the internal core diameter. By analyzing Fig. 6(d), the efficiency improves at different values of misalignment depending on the size of the inner core diameter. If it is too small, the efficiency worsens even with misalignments smaller than $10 \mathrm{~mm}$. Observe that, when the inner diameter is $20 \mathrm{~mm}$, the TET reduces its efficiencies in about $40 \%$ by increasing its misalignment from 5 to $10 \mathrm{~mm}$. This shows that the inner core diameter is determinant in the stability of the TET.

Different than the efficiency, in most of the cases, the relative current density deteriorates greatly with small misalignments of approximately $5 \mathrm{~mm}$ and then varies slightly with bigger misalignments, even improving at larger misalignments. It is not easy to find a pattern on the behavior of the relative current density. However, Fig. 6 shows that for misalignment of $17.5 \mathrm{~mm}$, the relative current density is extremely high - the highest for most of the simulated configurations. Despite this value of misalignment, observe that the variation on the relative current density follows the same trend independent of the number of turns in the internal and external coils, frequency, and central core diameter. This similar trend cannot be observed when varying the inner and outer core diameter. Indeed, Fig. 6(d) and Fig. 6(e) show different curves following different trends depending on the size of the inner and outer core diameter.

This behavior can be understood by analyzing the chosen geometry at some specific misalignment positions. For better perception, Fig. 7 shows the qualitative effect of the induced current density in the skin for the default values of the dimensions when the coils are aligned and misaligned at the specific positions where the trend changes $-10 \mathrm{~mm}, 17.5 \mathrm{~mm}$ and $30 \mathrm{~mm}$. 
(a)
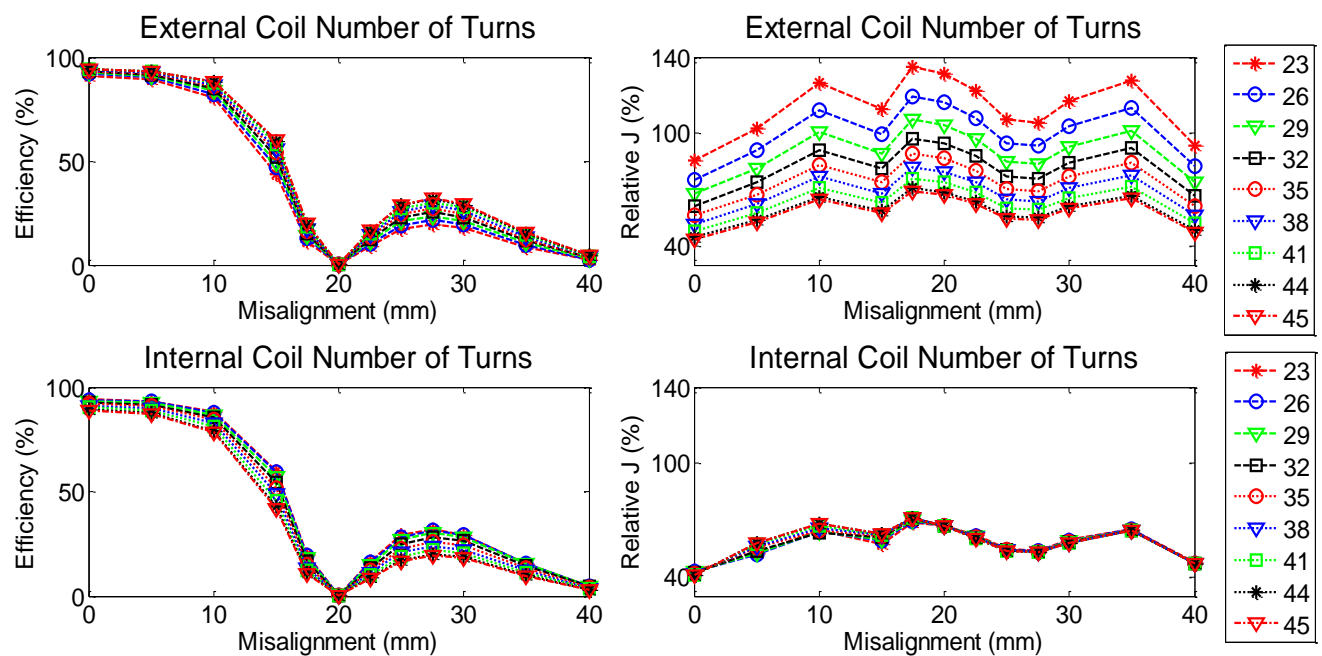

(c)
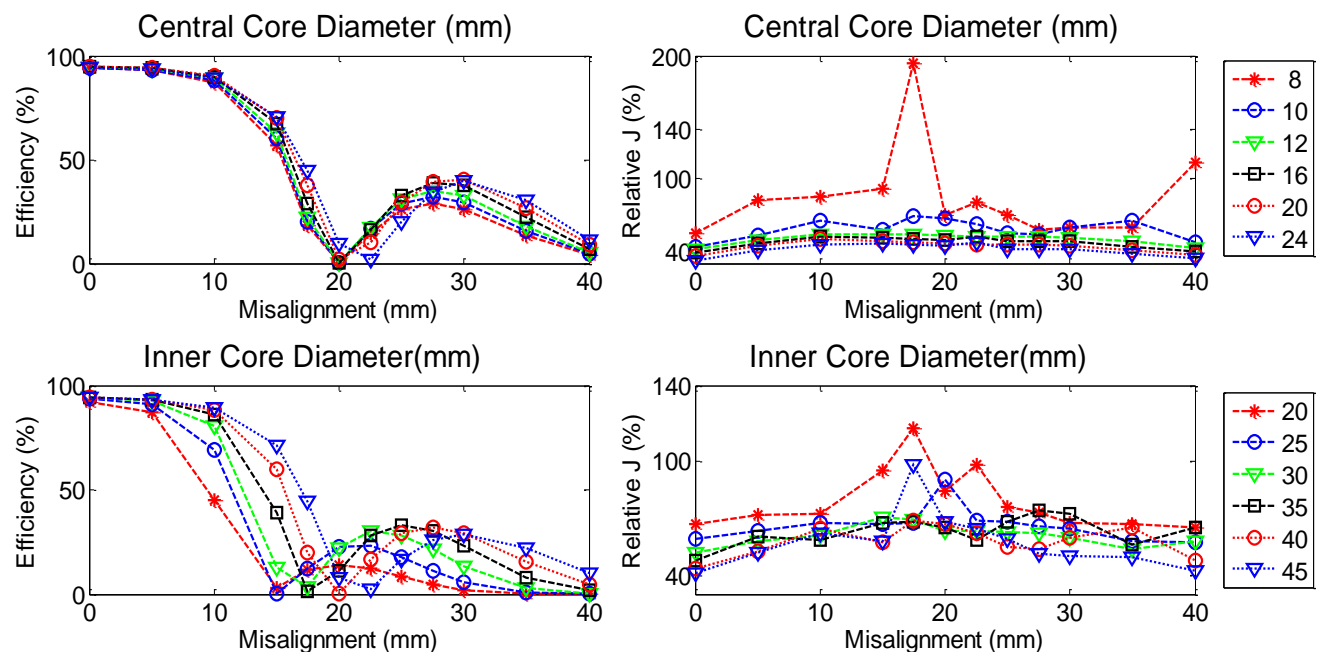

(e)
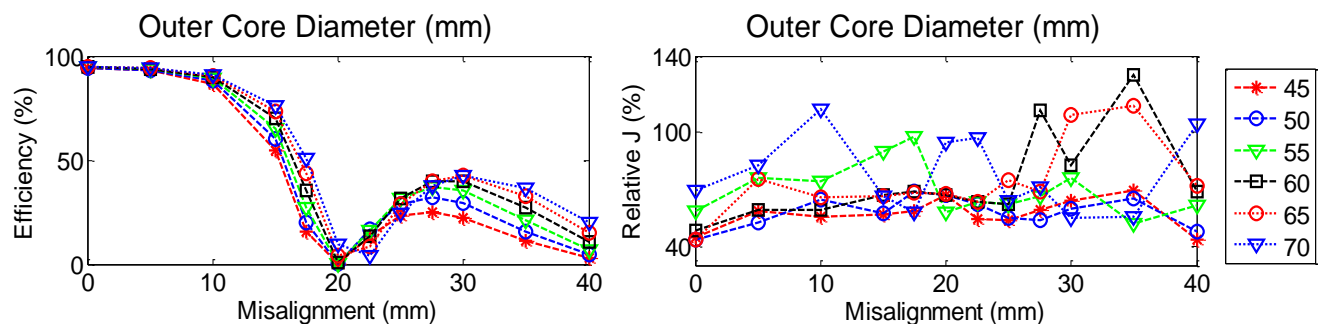

Frequency $(\mathrm{kHz})$
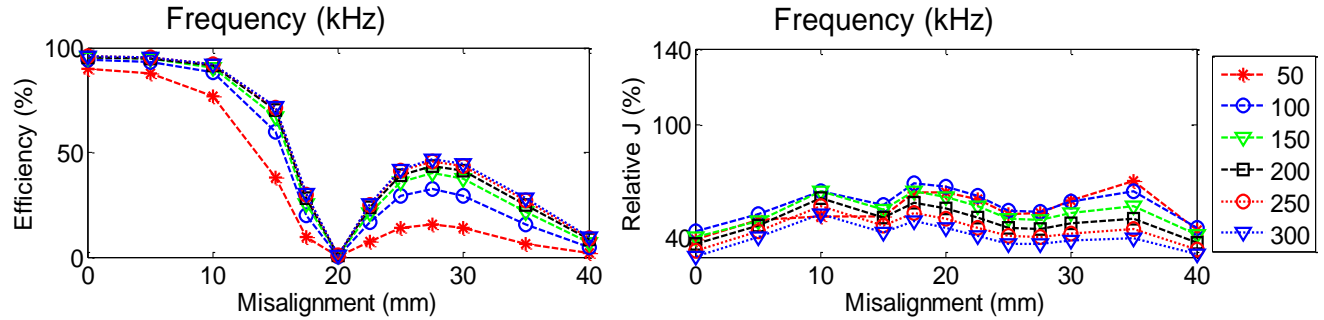

Fig. 6. Response of TET to the misalignment between coils at each configuration. (external coil $=45$ turns; internal coil $=23$ turns).

Observe that, when the coils are aligned, it makes the best coupling for this application, thus lower induced current and higher efficiency are expected. When the misalignment starts, the coupling starts 
weakening thus worsening the efficiency and the current density induced in the skin. When it is just after $10 \mathrm{~mm}$, the external core has just finished facing the internal core, as shown in Fig. 7(b). For this reason, it is expected that the efficiency and relative current density at the skin deteriorate more by increasing this misalignment.

Nevertheless, as the central part of the primary core loses connection with the central part of the secondary core, it gets closer to make a better coupling with the outside edges (defined by the area between the inner and outer diameter). When the misalignment is close to $17.5 \mathrm{~mm}$ the central part begins to align with the outside edges, as shown in Fig. 7(c). Thus, the TET start having a different new magnetic circuit with smaller reluctance and consequently, an improvement in the TET's performance is expected as can be observed in Fig. 6 and Fig. 7.

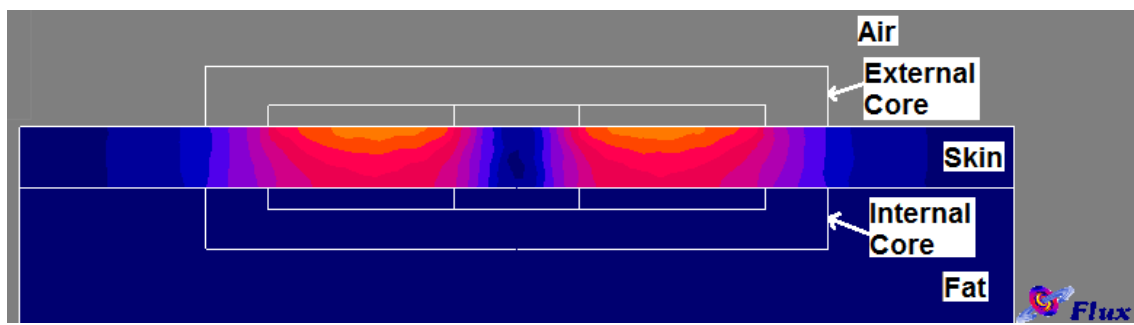

(a) $0 \mathrm{~mm}$

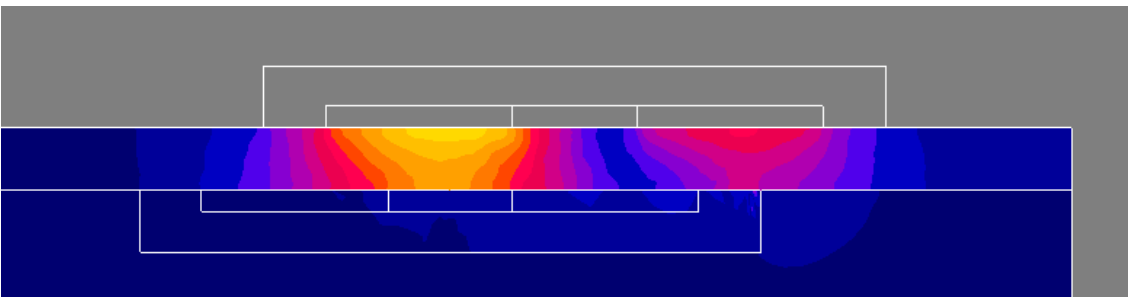

(b) $10 \mathrm{~mm}$
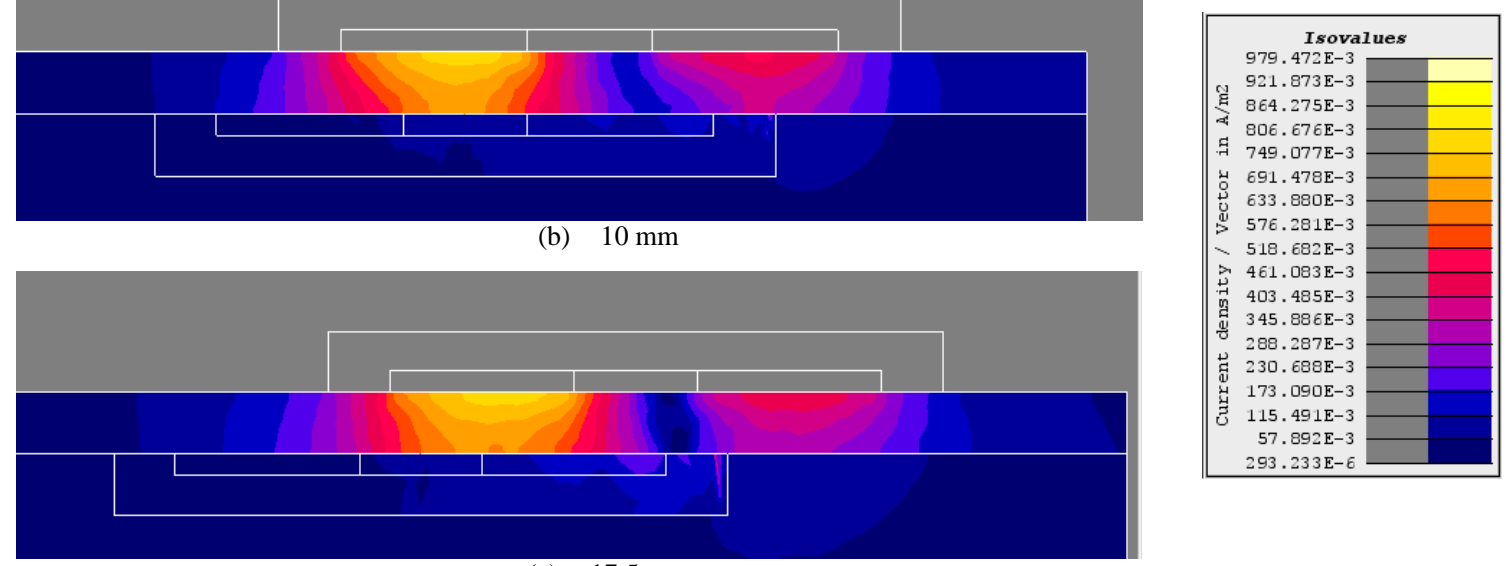

(c) $17.5 \mathrm{~mm}$

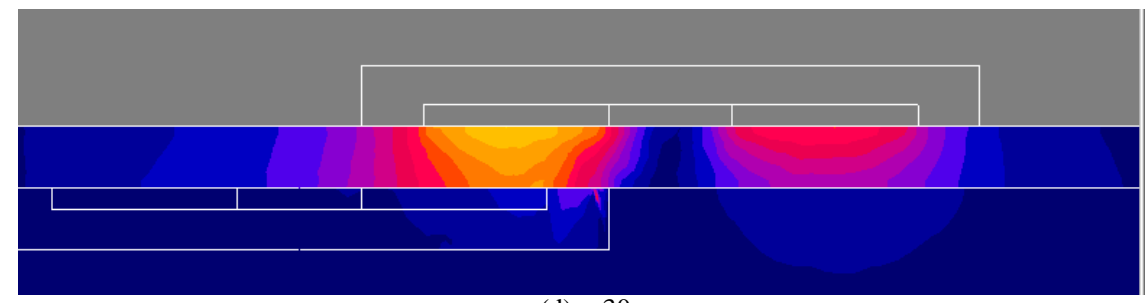

(d) $30 \mathrm{~mm}$

Fig. 7. Isovalues of the Induced Current Density when the coils are with different misaligning situations, such as (a) $0 \mathrm{~mm}$; (b) $10 \mathrm{~mm}$; (c) $17.5 \mathrm{~mm}$; (d) $30 \mathrm{~mm}$. TET with 45 turns in the primary supplied by $24 \mathrm{~V}$ at $100 \mathrm{kHz}$ and 23 turns in the secondary connected to a resistance of $18.75 \Omega$. Primary and secondary coils contain core type I with relative permeability $3000,10 \mathrm{~mm}$ in the central core diameter, $40 \mathrm{~mm}$ in the inner diameter and $50 \mathrm{~mm}$ in the outer diameter.

While the central part of the primary core is aligned with any magnetic part of the secondary core, the values of the efficiency should maintain similar values, depreciating again when the primary loses 
magnetic coupling with the secondary, what happens at $30 \mathrm{~mm}$. This explanation also justifies why the worst values of relative current density occur when the internal core diameter is smaller than 30 $\mathrm{mm}$ or larger than $45 \mathrm{~mm}$, what can be observed in Fig. 6 by analyzing the performance of the TET with the change of the internal core diameter.

It is noteworthy to mention that the value of the current density is exceeding the limit established by ICNIRP when the coils are misaligned only for some configurations, mainly when the external coil has less than 32 turns.

Moreover, it can be concluded that the outer core diameter should not be larger than $55 \mathrm{~mm}$, because such configurations entail in higher relative current density, even though better values are achieved when the misalignment is $17.5 \mathrm{~mm}$. It is noteworthy to remember that, these analyzes are being performed only for core type I, as mentioned in the beginning of this section.

In addition to this analysis with geometric dimensions, this paper also studied the sensitivity of the efficiency and induced current density at configurations with different core permeability in the range described by Table I. Fig. 8 shows this result.

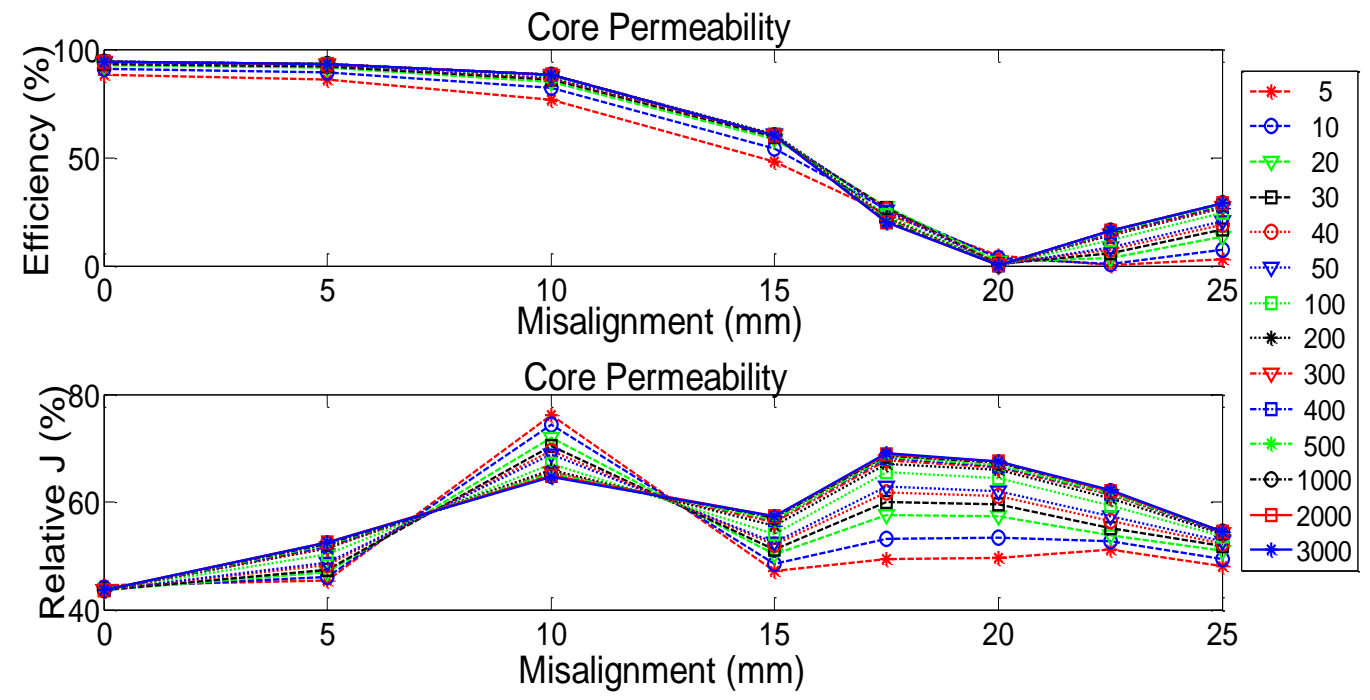

Fig. 8. Response of TET to the misalignment between coils at each simulated permeability. (TET with 45 turns in the primary supplied by $24 \mathrm{~V}$ at $100 \mathrm{kHz}$ and 23 turns in the secondary connected to a resistance of $18.75 \Omega$. Primary and secondary coils contain core type I with relative permeability $3000,10 \mathrm{~mm}$ in the central core diameter, $40 \mathrm{~mm}$ in the inner diameter and $50 \mathrm{~mm}$ in the outer diameter.).

From Fig. 8, the efficiency changes with the misalignment in the same way for core relative permeability above 30 . For core permeability smaller than 30 , the sensitivity of the efficiency with respect to the misalignment is slightly different, affecting more the efficiency at misalignments higher than $20 \mathrm{~mm}$.

Regarding the relative induced current density, it has the highest variations with the misalignment as the core permeability has the lowest values. This means that the lower the core permeability is, the more sensitive the induced current density is to misalignments. 


\section{CONCLUSIONS}

This paper made a comparison among the performances of different types of core geometry of TETs and realized that the use of round core with the flat coil inserted in the core, type I, brings the best efficiency at the lowest values of relative induced current density, what is one of the constraints for a safe use of the device.

This conclusion was also observed when comparing the different geometries with different permeability of the material, what makes this type of geometry possible to be built even with materials that are not Ferrite, such as plastic ferrites or magnetorheological fluids [10].

In addition, this paper made a more thorough analysis in this recommended option, investigating the behavior of this configuration in different sizes and for different positions of misalignment between the coils. It could be observed that:

- The stability of the efficiency is strongly dependent on the inner core diameter, getting worse as the diameter gets smaller.

- In order not to exceed the limits of the induced current density in the skin in the analyzed cases, the default external coil must have more than 32 turns if the internal core has 23 turns.

- The core relative permeability does not affect much the efficiency for values above 50, but the induced current density is as sensitive to misalignments as the core relative permeability is lower. Thus, the use of new materials with core relative permeability as low as 50 is acceptable in this case.

The performance of the TET with misalignments were detailed, explaining the reasons of change in the trend at some specific positions and indicating that this particular TET should not be misplaced by more than $15 \mathrm{~mm}$.

\section{ACKNOWLEDGMENT}

The authors would like to thank Prof. Florent Morel from the Ecole Centrale de Lyon for his revision and suggestions. Moreover, the authors would like to thank the support from Sao Paulo Research Foundation (FAPESP) under grant 2011/18341-3 and National Council of Scientific and Technologic Development of Brazil, CNPq, under grant 309350/2012-3/PQ.

\section{REFERENCES}

[1] J. Ma, Q. Yang, and H. Chen, "Transcutaneous Energy and Information Transmission System with Optimized Transformer Parameters for the Artificial Heart", IEEE Transactions On Applied Superconductivity, vol. 20, no. 3, pp. 798-801, 2010.

[2] H. Miura, S. Arai, Y. Kakybari, F. Sato, H. Matsuki, and T. Sato, "Improvement of the transcutaneous energy transmission system utilizing ferrite cored coils for Artificial Hearts", IEEE Transactions On Magnetics, v.42, no. 10, pp. 3578-3580, 2006.

[3] H. Matsuki, M. Shiiki, K. Murakami, and T. Yamamoto, "Investigation of Coil Geometry for Transcutaneous Energy Transmission for Artificial Heart", IEEE Transactions on Magnetics, vol. 28, no. 5, pp. 2406-2408, 1992.

[4] G. E. Miller, “Artificial Organs" (VA, USA. Morgan \& Claypool publishers, 2006).

[5] C. M. Zierhofer, and E. S. Hochmair, "Geometric approach for coupling enhancement of magnetically coupled coils", IEEE Transactions on Biomedical Engineering, vol. 43, no. 7, 1996.

[6] Institute for Applied Physics Nello Carrara. An Internet resource for the calculation of the dielectric properties of the body tissues in the frequency range $10 \mathrm{~Hz}-100 \mathrm{GHz}$. http://niremf.ifac.cnr.it/tissprop/.

[7] K. Shiba, M. Nukaya, T. Tsuji and K. Koshiji, "Analysis of Current Density and Specific Absorption Rate in Biological Tissue Surrounding Transcutaneous Transformer for an Artificial Heart", IEEE Transactions on Biomedical Engineering, 45(1), 2008, 205-213. 
[8] ICNIRP (International Commission on Non-Ionizing Radiation Protection), "Guidelines for Limiting Exposure to Time-Varying Electric, Magnetic, and Electromagnetic Fields (up to $300 \mathrm{GHz}$ )". Health Physics, 74(4), 1998, 494-522.

[9] J. Jin, "The Finite Element Method in Electromagnetics", John Wiley \& Sons, Inc., 2nd edition, 2003.

[10] T. M. Simon, F. Reitich, M. R. Jolly, K. Ito, H. T. Banks, "Estimation of the Effective Permeability in Magnetorheological Fluids", Mathematical and Computer Modelling, 33 (1-3), pp. 273-284, 2001. 